\title{
Measles and rubella elimination in the Eastern Mediterranean Region: successes and challenges
}

Nadia Teleb, ${ }^{1}$ Hoda Atta ${ }^{2}$ and Rana Hajjeh ${ }^{3}$

${ }^{1}$ Regional Adviser, Vaccine Preventable Diseases and Immunization Programme, Department of Communicable Diseases; ${ }^{2}$ Acting Director, Department of Communicable Diseases; ${ }^{3}$ Director of Programme Management, World Health Organization Regional Office for the Eastern Mediterranean, Cairo, Egypt. (Correspondence to: Nadia Teleb: telebn@who.int).

Citation: Teleb N; Atta H; Hajjeh R. Measles and rubella elimination in the Eastern Mediterranean Region: successes and challenges. East Mediterr Health J. 2019;25(10):667-668. https://doi.org/10.26719/2019.25.10.667

Copyright (C) World Health Organization (WHO) 2019. Some rights reserved. This work is available under the CC BY-NC-SA 3.o IGO license (https:// creativecommons.org/licenses/by-nc-sa/3.o/igo).

In 2019, upon reviewing countries' reports, the regional verification commission for measles and rubella elimination declared elimination of measles and rubella in Bahrain, Islamic Republic of Iran and Oman. This achievement in the Eastern Mediterranean Region (EMR) constitutes a major success, since it occurs at a time when there remain highly challenging situations in several countries of the Region. In addition, there are unprecedented high transmission rates of measles throughout the world, with other World Health Organization (WHO) regions either not progressing (status quo) or regressing. Indeed, the WHO Region of the Americas and several other countries have in fact lost the status of measles elimination $(1,2)$.

In 1997, the countries of the EMR adopted measles elimination as a goal to be achieved by 2010 (3). Due to delays the goal was postponed to 2015 (4) and then to 2020 as part of the Eastern Mediterranean Vaccine Action Plan (5). While there was no official goal for rubella elimination, several countries in the Region have established a national rubella elimination goal as part of an integrated measles and rubella elimination programme.

The regional strategies for achieving measles elimination, adapted from the Global Measles and Rubella Strategic Plan 2012-2020 (6), includes achieving high population immunity through a vaccination coverage of at least $95 \%$. This is implemented with 2 doses of measles-containing vaccine in all districts through routine immunization, complemented by supplementary immunization activities (SIAs) where needed. In addition, high quality measles and rubella surveillance is conducted and supported by national proficient laboratories that facilitate optimal outbreak response, including case management, advocacy and communication.

Countries of the EMR have implemented the regional strategies for measles elimination with variable levels of success. In 2018, estimated coverage with first dose of measles-containing vaccine $(\mathrm{MCV} 1)$ was $95 \%$ or above in 11 countries, $90-94 \%$ in 2 countries and below 90\% (range $46-88 \%$ ) in 9 countries (7). In the same year, among the 21 countries with a routine schedule of 2 or more doses of measles-containing vaccine (MCV2), estimated coverage was $95 \%$ or more in 12 countries, $90-94 \%$ in 1 country and below 90\% (range $39-81 \%$ ) in 8 countries (7). In 2018, among the 17 countries that have introduced rubellacontaining vaccine, estimated coverage with first dose of rubella-containing vaccine $(\mathrm{RCV} 1)$ was $95 \%$ or above in 12 countries, $90-94 \%$ in 1 countries and below 90\% (range $63-81 \%$ ) in 4 countries (7).

To supplement routine immunization coverage and boost population immunity, more than 670 million people aged from 6 months to 60 years were vaccinated through 257 national or subnational SIAs during the period 2000-2019 (6).

Measles and rubella case-based surveillance, supported by a network of WHO accredited national measles and rubella laboratories and 3 regional and subregional reference laboratories, was established in all EMR countries, except Djibouti and Somalia. Measles surveillance system performance indicators show that the majority of the low-incidence countries are meeting the elimination surveillance standards (8).

Substantial progress has been made since the EMR countries first resolved to eliminate measles. In 2018, 7 countries reported endemic measles virus transmission of less than 1 case per million population (range 0.0-0.7 per million population) (9). Moreover, between 2000 and 2017, more than 2.5 million deaths have been averted in the EMR thanks to vaccination (10).

To verify achieving the elimination goal, a Regional Verification Commission was nominated in February 2018 by the Regional Director for the WHO Regional Office for the Eastern Mediterranean. Implementation of the verification process started immediately thereafter, whereupon the national verification committees and their secretariat in 12 Member States (low-incidence countries) were briefed on documentation of elimination of measles and rubella as well as developing countries' reports and requests for verification of elimination.

The achievement of measles and rubella elimination status in three EMR countries, especially in the Islamic Republic of Iran - a vast populous country that is facing several challenges - is expected to increase visibility of this goal and encourage other countries to take up the challenge. Efforts should focus on increasing routine 
MCV1 and MCV2 vaccination coverage and ensuring that routine immunization services and SIAs reach atrisk populations that reside in areas with poor access to vaccination services. Follow-up SIAs, whether national or targeted subnational, need to be conducted periodically until routine coverage of over $95 \%$ with both MCV1 and $\mathrm{MCV} 2$ is achieved and maintained in every district. Conducting SIAs in conflict settings and in areas with no local government requires strong partnership and close links with local communities. Monitoring and strengthening measles surveillance performance will help rapidly identify and characterize outbreaks, guide response activities and provide evidence for refining measles elimination strategies, in addition to providing evidence for achieving elimination.

There is a dire need to further strengthen the immunization system and achieve universal immunization coverage, as part of expanding primary health care services and achieving universal health coverage, in order to reach every child with vaccination and leave no-one behind. The crucial need for ensuring high measles vaccination coverage, and provision of measles vaccine in emergency settings to protect people from measles epidemics, should always be part of preparedness and response to health emergencies. Strengthening national immunization decision-making capacity, along with building strong independent National Immunization Technical Advisory Groups (NITAGs) that guide immunization-related decision and monitor implementation of a sound immunization programme, is highly required. It is imperative to strengthen human resource capacity, especially management capacity, at different levels of the immunization programme in order to support programme expansion and effectively implement sound immunization strategies and achieve various immunization goals, including measles elimination.

\section{References}

1. World Health Organization. Measles outbreaks continue to spread rapidly around the world. Immunization, vaccines and biologicals. Geneva: World Health Organization; 2019 (https://www.who.int/immunization/newsroom/new-measles-data-august-2019/en/, accessed 23 October 2019)

2. Fore HH, Ghebreyesus TA. Measles cases are up nearly 300\% from last year. This is a global crisis. CNN Online, 15 April, 2019 (https://edition.cnn.com/2019/04/15/opinions/measles-cases-rise-global-crisis-fore-ghebreyesus/index.html, accessed 23 October 2019).

3. Forty-fourth Session of the Regional committee for the Eastern Mediterranean, Tehran, Islamic Republic of Iran, 4-7 October 1997. Elimination and eradication of diseases, with special reference to measles and tuberculosis (EM/RC44/R.6). (http://apps. who.int/medicinedocs/documents/s17706en/s17706en.pdf).

4. Fifty-eighth Session of the Regional Committee for the Eastern Mediterranean, Cairo, Egypt, 2-5 October 2011. Scaling up expanded programme on immunization to meet global and regional targets (EM/RC58/R.5) (http://applications.emro.who.int/docs/ RC_resolutions_2011_r5_13972.pdf?ua=1).

5. Sixty-second Session of the Regional Committee for the Eastern Mediterranean, Kuwait, 5-8 October 2015. Annual report of the regional director 2014 (EM/RC62/R.1) (http://applications.emro.who.int/docs/RC62_Resolutions_2015_R1_16570_EN.pdf?ua=1).

6. World Health Organization. Global measles and rubella strategic plan: 2012-2020. Geneva: World Health Organization; 2012 (http://www.who.int/immunization/documents/control/ISBN_978_92_4_150339_6/en/).

7. World Health Organization \& United Nations Children's Fund (UNICEF). Estimates of national immunization coverage: immunization, vaccines and biologicals: data, statistics and graphics. Geneva: World Health Organization; 2019 (http://apps.who.int/ immunization_monitoring/globalsummary/timeseries/tswucoveragebcg.html, accessed 17 October 2019)

8. World Health Organization. Retrospective measles data on supplementary immunization activities - immunization, vaccines and biologicals: data, statistics and graphics. Geneva: World Health Organization; 11 September 2019 (https://www.who.int/ immunization/monitoring_surveillance/data/Summary_Measles_SIAs.xls?ua=1, accessed 17 October 2019)

9. World Health Organization Regional Office for the Eastern Mediterranean. Monthly reported measles and rubella case-based surveillance data. Vaccine preventable diseases and immunization: measles monthly bulletin, cumulative report for 2018. Cairo: World Health Organization; 2019. (http://www.emro.who.int/vpi/publications/measles-monthly-bulletin.html, accessed 17 October 2019).

10. Weekly Epidemiological Record (WER). Progress towards regional measles elimination - worldwide, 2000-2017. Weekly Epidemiological Record (WER), 30 November 2018; 93:649-660 (https://apps.who.int/iris/bitstream/handle/10665/276217/WER9348. pdf). 